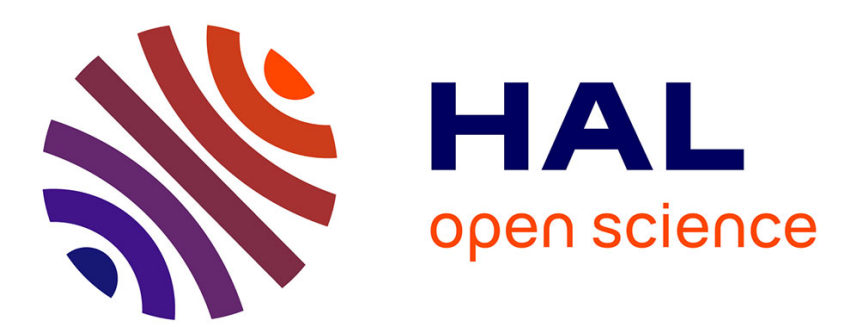

\title{
Adhesion induced non-planar and asynchronous flow of a giant vesicle membrane in an external shear flow
}

Cyrille Vézy, Gladys Massiera, Annie Viallat

\section{To cite this version:}

Cyrille Vézy, Gladys Massiera, Annie Viallat. Adhesion induced non-planar and asynchronous flow of a giant vesicle membrane in an external shear flow. Soft Matter, 2007, 3, pp.844. 10.1039/b700550d . hal-00321742

\section{HAL Id: hal-00321742 \\ https://hal.science/hal-00321742}

Submitted on 15 Sep 2008

HAL is a multi-disciplinary open access archive for the deposit and dissemination of scientific research documents, whether they are published or not. The documents may come from teaching and research institutions in France or abroad, or from public or private research centers.
L'archive ouverte pluridisciplinaire HAL, est destinée au dépôt et à la diffusion de documents scientifiques de niveau recherche, publiés ou non, émanant des établissements d'enseignement et de recherche français ou étrangers, des laboratoires publics ou privés. 


\title{
Adhesion induced non-planar and asynchronous flow of a giant vesicle membrane in an external shear flow
}

\author{
Cyrille Vézy $^{a}$, Gladys Massiera ${ }^{b}$ and Annie Viallat ${ }^{a}$
}

5

We show the existence of a flow at the surface of strongly adhering giant lipid vesicles submitted to an external shear flow. The surface flow is divided into two symmetric quadrants and present two stagnation points (SP) on each side of the vesicle meridian plane. The position of these stagnation points highly depends on the adhesion strength, characterized by the ratio of the contact 10 zone diameter to the vesicle diameter. Contrary to the case of non adhesive vesicles, streamlines do not lie in the shear plane. By avoiding the motionless contact zone, streamlines result in threedimensional paths, strongly asymmetric away from the SP. Additional shearing dissipation may occur on the membrane surface as we observed that the mean rotational velocity of the membrane increases towards the vesicle SP, and is mainly determined by the adhesion induced vesicle shape.

\section{${ }_{15}$ Introduction}

Situations, where soft deformable objects with a fluid interface like drops, vesicles and cells are subjected to hydrodynamics flows at low Reynolds numbers, are involved in an increasing number of engineering and biophysical ${ }_{20}$ processes including microfluidics and emulsion processing ${ }^{1}$, biomechanics $^{2}$ and blood circulation ${ }^{3,4}$. Numerous studies have brought insights into the specificity of the motions of this class of particles under simple Poiseuille or shear flows. In particular, it is well known that under simple shear flow, 25 the fluidity of the particle interface allows surface flows, which are at the origin of a typical motion called tanktreading. This regime of motion, observed under suitable conditions of viscosity, aspect ratio of the particle and shear rate $^{5-7}$, is characterized by a stationary shape of the particle, a steady 30 finite inclination angle with respect to the flow direction and the rotation of the surface layer or of the membrane of the particle about its centre of mass.

The surface flow of the incompressible membrane of tanktreading red blood cells has long been observed from the 35 position of small latex beads stuck onto the rotating membrane ${ }^{4}$. Similarly, the motion of lipid vesicle membranes has been reported for tanktreading vesicles in shear flow ${ }^{8,9}$ and for vesicles sliding along an inclined plane in a quiescent fluid ${ }^{10}$ by tracking small lipid defects linked to the membrane.

40 It was observed both for red blood cells biconcave disks and spherical or ellipsoidal vesicles that all elements of the membrane flow on closed surface streamlines, which describe quasi-planar orbits in the shear plane $e^{4,10}$. The surface flow of the red blood cell membrane, which is bound to a solid elastic 45 cytoskeleton is synchronous in the sense that the cyclic motions along the streamlines have one common frequency, the tanktreading frequency ${ }^{4}$. It means that adjacent membrane elements remain adjacent after a full revolution along the streamlines. The revolution velocity on the streamlines of 50 vesicle surfaces has not been experimentally explored in detail. A short report on a non spherical vesicle fixed by a thin lipid tube to the substrate suggests that the angular velocity along the surface streamlines of the viscous membrane, depends on the lateral streamline position with respect to the 55 meridian orbit of the vesicle ${ }^{11}$.

The theoretical problem of the surface flow of a tanktreading fluid membrane with fixed area and bending rigidity in shear flow is complex. Using a simple surface velocity field and under the hypothesis of a synchronous 60 motion along planar elliptical orbits, Keller and Skalak ${ }^{12}$ analytically derived the equation of motion of a fluid ellipsoid and computed the tanktreading velocity. Secomb and Skalak ${ }^{13}$ generated more realistic area-conserving velocity fields on the surface of ellipsoids. In this case, they showed that the motion 65 along streamlines which presented a curvature towards each end of the cell resulted in less dissipation than the motion with planar orbits. More recently, Kraus et al. ${ }^{14}$ proposed a numerical approach to deal with an incompressible membrane with bending rigidity and an ellipsoidal shape of arbitrary 70 aspect ratio. He showed that the streamlines lie in a plane parallel to the shear plane and that when the vesicle shape departs markedly from the spherical shape the revolution velocity varies along the contour in a way that velocities are smaller towards the poles of the vesicle. Very recent 75 theoretical works numerically dealt with the full problem of motion of vesicles, including the effects of thermal fluctuations, but they did not focus on surface flows ${ }^{15}$.

Contrarily to the case of non-bounded shear flows, the surface flow of vesicles firmly adhered to a plane wall in a 80 shear flow has not generated much experimental studies ${ }^{9,16}$, although the question is of interest in terms of mobility and recruitment of cell membranar receptors towards the adhesive zone on the subtrate, which bear associated ligands ${ }^{17}$. Two theoretical works describe the motion of bound vesicles under 85 flow 1617 focusing their studies on the unbinding of nonspecifically adhered vesicles, but do not describe the membrane flow. The main questions we want to address in this paper are: is the vesicle membrane able to flow in presence of attractive interaction with the substrate, either 90 induced by the binding of specific membranar linkers or by a 
non specific adhesive field? What are the characteristics of surface streamlines and associated velocity field? How do these features vary with the vesicle size and the contact area?

The paper is organized as follows. After the description of 95 materials and methods, we present the observation of vesicles shape and surface flows under shear flow. We characterize the streamlines on the vesicle surface as a function of the relative size of the contact zone with the substrate. Then, we determine the variation of the angular velocity along a 100 streamline with its position on the surface. We finally shortly discuss the obtained results.

\section{Experimental}

\section{Substrates}

Two types of substrate are prepared in order to induce either 105 non specific adhesion (charged coverslips covered by poly-Llysin) or specific adhesion (coverslips covered by histidine ligands). All products were purchased from Sigma except the Histidine peptide (KGGSGGSYGGSGGSHHHH-HH) obtained from Covalab (Villeurbanne, France). Adhesion is 110 induced by chelation between two histidin groups and a Nickel ion. The stability constant ${ }^{18}$ of the complex $\mathrm{C}^{*}$, $\mathrm{K}=\left[\mathrm{C}^{*}\right] /[\mathrm{Ni}][\mathrm{Hist}]^{2}$, is equal to $10^{15.9}$.

Prior to any chemical treatment, glass coverslips are cleaned by immersion in a $14.5 \mathrm{M} \mathrm{NaOH}$ solution for 5 115 minutes and rinsed extensively with pure water. Polylysin coverslips are obtained by incubation in a polylysin solution $\left(10^{-3} \% \mathrm{v} / \mathrm{w}\right)$ for $30 \mathrm{~min}$, followed by rinsing with ultrapure water. The histidine functionalization is obtained according to the following process: a glutaraldehyde peptide is first bound 120 to the silane activated glass surface by reaction between an aldehyde bond on the peptide and an amine bond on the silane. The histidine peptide is then bound to the glutaraldehyde peptide via its terminal amine function. The 6histidine peptide was chosen since it maximizes the ${ }_{125}$ probability of chelation of a $\mathrm{Ni}$ on a vesicle ${ }^{19}$. Technically, the glass slides receive sequentially the following treatments at room temperature: incubation in a silane solution $(1 \% \mathrm{v} / \mathrm{v} 3-$ aminopropyl-tri-ethoxysilane and $5 \mathrm{mM}$ acetic acid in water) for 20 minutes under gentle mixing, rinsing with pure water,

130 curing for $15 \mathrm{~min}$ at $100{ }^{\circ} \mathrm{C}$, incubation in a glutaraldehyde solution $(10 \% \mathrm{w} / \mathrm{w})$ for $90 \mathrm{~min}$ under gentle mixing. Coverslips are then incubated for 2 hours at $37^{\circ} \mathrm{C}$ in a solution of histidine peptide $(1.2 \mathrm{mg} / \mathrm{ml})$ diluted in a carbonate/bicarbonate buffer $(0.2 \mathrm{M}, \mathrm{pH}=8)$. After rinsing 135 with a PBS-Triton solution (1\%), coverslips are incubated for 1 hour at $37^{\circ} \mathrm{C}$ in an ethanolamine solution $(0.2 \mathrm{M}$ at $\mathrm{pH}=10)$ in order to passivate the substrates and to prevent non specific adhesion. The coverslips are rinsed again using the PBSTriton solution. Finally, the substrates are incubated in a 140 sodium borohydre $\left(\mathrm{NaBH}_{4}\right)$ solution $(3.5 \mathrm{mg} / \mathrm{ml})$ at room temperature for 1 hour without shaking, to reinforce the bond between the glutaraldehyde and the peptide (reduction of the the imine group of the glutaraldehyde / peptide bond into an amine group). Coverslips can then be stored up to 8 days at ${ }_{145} 4^{\circ} \mathrm{C}$ in a sodium azide PBS buffer $\left(0.1 \% \mathrm{NaN}_{3} \mathrm{v} / \mathrm{v}\right)$.

Vesicles
The lipids, 1,2-dioleoyl-sn-glycero-3-phosphocholine (DOPC) and 1,2 - Dioleoyl - sn - Glycero - 3 - \{[N (5 - Amino - 1 Carboxypentyl)iminodi Acetic Acid] Succinyl $\}$ (Nickel Salt) 150 (DOGS-NTA-Ni) are purchased as a powder from Avanti Polar Lipids, and diluted at $2 \mathrm{mg} / \mathrm{ml}$ in a chloroform/methanol solution (9:1). Giant unilamellar lipid vesicles were prepared either from pure DOPC or from a 95:5 mixture of DOPC and DOGS-NTA-Ni, using the standard electroformation 155 method $^{20}$. Sucrose solutions $(50 \mathrm{mM}$ and $200 \mathrm{mM})$ are used as the internal fluid. Vesicles are then diluted in a solution of glucose with the same osmolarity as the internal solution of sucrose and is prepared in a HEPES buffer (10 mM, pH 8).

\section{Microscopy}

160 'Side view' observation was obtained by orienting a phase contrast inverted microscope (Leica DM IRB, Wetzlar, Germany) horizontally and by working at low-angle incidence. The observation plane is then parallel to gravitational force and to the shear plane ${ }^{8}$. It provides well165 defined side-view images of both vesicles and their reflections on the substrate. Most observations were performed with a phase contrast $20 \mathrm{X}$ objective (long working distance). Pictures were captured with a CCD (Cohu) and a dvcam video-recorder (Sony, DSR-25).

170 Reflection interference contrast microscopy (RICM) was used to determine the contact area of the adhered vesicle with the substrate ${ }^{21}$. This microinterferometric technique generates an intensity pattern by interference of two reflected rays: the incident light reflected both from the glass-buffer interface 175 and from the buffer-vesicle interface. Strongly bound parts of the membrane appear as dark regions on the characteristic RICM patterns. Both standard "bottom view" observation and RICM observation were performed using a phase contrast inverted microscope (Olympus, IX71) equiped with an 180 antiflex objective (Plan Neofluar, x63 /1.25 Oil, Zeiss). Epiillumination through the objective was rendered monochromatic by passing light from a $100-\mathrm{W} \mathrm{Hg}$ vapor lamp (Olympus) through an interference filter (Ifg $546.1 \mathrm{~nm}$ ).

\section{Flow}

185 For side view observation, the flow chamber was a parallelepiped-plate chamber (spectrophotometric circulation chamber from Hellma (Müllheim), height $=1 \mathrm{~mm}$, width $=10$ $\mathrm{mm}$, length $=45 \mathrm{~mm}$ ) with four optical faces. At one end, a rectangular opening give us the possibility to introduce a 190 coverslip. The laminar shear rate (from 1 to $12 \mathrm{~s}^{-1}$ ) was applied with $20 \mathrm{~mL}$ syringe mounted on a syringe pump. Shear rate calibration was performed by measuring the translational speed of polystyrene beads $(2 \mu \mathrm{m}$ of diameter $)$ versus their distance from the substrate. For bottom view 195 experiments, the chamber was a plexiglas block bearing a cavity of $15.9 \times 0.99 \times 0.9 \mathrm{~mm}^{3}$ surrounded by a toric gasket. Histidine or polylysin coverslips are maintained against the gasket with a screwed steel plate.

\section{Protocol of measurement}

Measurements were performed at a distance from the substrate less than $50 \mu \mathrm{m}$, so that the shear rate can be taken as constant 
and equal to the wall shear rate. All vesicles studied were at a minimal distance of $1 \mathrm{~mm}$ from the nearest optical face of the 205 chamber, so that velocity field and wall shear rate values were not disturbed by the presence of the side walls. Vesicles are slowly injected in the chamber and allowed to settle. One well contrasted axisymmetric vesicle is chosen at rest. A flow is then applied. The wall shear rate applied to the vesicle is then 210 increased gradually from $1.3 \mathrm{~s}^{-1}$ to $11 \mathrm{~s}^{-1}$, by increasing step by step the flow rate in the chamber.

\section{Image analysis}

For side view image analysis, we developed an IDL (RSI) code to determine the vesicle contour. Both the vesicle and its

215 reflection are fit by a sphere, and their intersection determine the adhesion parameter (contact length $=\mathrm{L}$ ). The membrane defect position was either tracked using an IDL tracking code $\$$, or manually selected on each image using imageJ software, depending on the contrast.

\section{${ }_{220}$ Results and Discussion}

We first determine vesicle shapes. We then show the existence of surface flows in the part of the membrane which is not at the close vicinity of the substrate. We then determine the surface streamlines and the angular velocity on the 225 streamlines.

All coordinates are indicated by using a Cartesian referential: $\mathrm{x}=$ flow direction; $\mathrm{y}=$ shear direction.

\section{Determination of vesicle shapes}

The projection of vesicles onto their meridian plane $(\mathrm{z}=0)$ is 230 directly observed from side-view microscopic imaging (Fig.

1). Shapes are stable in time and do not significantly change upon increasing the shear rate. They are well-described by truncated spheres, when the shear rate is less than $12 \mathrm{~s}^{-1}$. It suggests that adhesion is strong, i.e., WA $>>\kappa$, with $\mathrm{W}$ the 235 adhesion energy per unit area, A the area of the adhesion disk and $\kappa$ the bending rigidity of the membrane. The shapes are characterized by their radius $\mathrm{R}$ and the position of the substrate $\left(\mathrm{y}=\mathrm{y}_{\mathrm{s}}\right)$ relatively to the sphere centre, which is taken as the origin of the referential. One directly derives the 240 diameter L of the contact zone in strong interaction with the substrate as shown in Fig. 1b. The full vesicle shape is determined by considering that the vesicles are axisymmetric about the y-axis.

The projection of vesicles onto their equatorial plane $(y=0)$ 245 is directly observed from regular "bottom view" phase contrast observation in the horizontal plane and yields vesicle radii (Fig. 2a). Moreover, simultaneous RICM observations show a strong interaction of the vesicle with the substrate over a large homogeneous disk of diameter L (Fig. 2b). There is no 250 interference fringes at the edge of the contact zone due to a sharp slope of the membrane relative to the substrate. Observation in the horizontal $(y=c s t)$ plane does not allow the full determination of the vesicle shape, since the vesicle axis of symmetry is perpendicular to the plane of observation.

${ }_{255}$ Based on side-view observations, we assume that the shape of these vesicles is also a truncated sphere.
Geometrical characteristics of studied vesicles are reported in Table 1 . The vesicles exhibit large contact area, $L / 2 \mathrm{R}$ values ranging from 0.46 to 1 (half-sphere). As all vesicles were 260 suspended in a solution of same osmolarity as that of vesicle inner solution, they were expected to be swollen and present a spherical shape. We therefore assume that upon adhering to the substrate the vesicles transiently opened several pores to release a part of the internal solution in order to relax the line 265 tension on the membrane ${ }^{22}$. It should be emphasized that the shape of these adhering vesicles, which consists of a circular disk like area on the substrate, a spherical cap on the opposite side, and a very small intermediate region of strong curvature along the contact line, reveals a large adhesion energy with 270 the substrate ${ }^{23}$.

\section{Surface flow observation}

We followed the movement of small defects (small clumps of lipids) bound to the vesicle membrane (approximate size $1 \mu \mathrm{m})$ under shear flow. We selected defects associated to the 275 inner part of the membrane, which may theoretically circulate on the inner side of the membrane even if its latter is at the immediate vicinity or in contact with the substrate.

The movement of these markers allows one to visualize the projection of surface motions. Sequences shown in Fig. 1 and ${ }_{280}$ Fig. 2 clearly demonstrate the existence of surface flows of the membrane, as already reported in ref 9 and 16 . The projection of the orbits described by the defects on the surface are determined by measuring their position as a function of time as shown in Fig. 1 and assumed to correspond to the 285 surface streamlines on the membrane. It is worth emphasizing that the Brownian motion of a defect is slow compared to the velocity at which it circulates on the membrane and does not perturb the determination of the surface streamlines. On a longer timescale, however, the defects slowly diffuse on the 290 membrane surface and explore various surface streamlines. The observation of the movement of a single defect therefore allows the determination of several orbits on a single vesicle.

The sequence in Fig. 2 shows that when the defect passes at the top of the vesicle membrane $(x=0)$, its orbit is quasi295 circular and lies in the plane $(\mathrm{z}=\mathrm{cst})$ parallel to the shear plane. When it approaches the substrate, as detected by the change of focus of the defect, its trajectory departs from the plane and avoids the contact zone. This zone and the water molecules that can possibly lie between the vesicle and the 300 substrate are therefore considered as being motionless.

\section{Streamlines determination}

The streamlines on each vesicle surface were obtained from the observation of a single defect. Its couple of coordinates ( $x$, $\mathrm{y})$ and $(\mathrm{x}, \mathrm{z})$ is directly measured from side view (projection 305 on the plane $z=0$ ) and regular bottom view (projection on the plane $y=0$ ) respectively. The third coordinate is calculated from the description of the vesicle as a truncated sphere of equation $\mathrm{x}^{2}+\mathrm{y}^{2}+\mathrm{z}^{2}=\mathrm{R}^{2}$ and $\mathrm{y}>\mathrm{y}_{\mathrm{s}}$. The projections of streamlines are illustrated on three vesicles in three planes (x, $310 \mathrm{z}),(\mathrm{x}, \mathrm{y})$ and $(\mathrm{z}, \mathrm{y})$ in Fig. 3. Data are either obtained from direct observation or from determination of one coordinate via the sphere equation. The three vesicles exhibit various aspect ratios, characterized by $\mathrm{L} / 2 \mathrm{R}$. Typical streamlines are 
illustrated in Fig. 3. The first feature to notice is that the 315 surface flow is divided into two symmetric quadrants observed on each side of the meridian plane $z=0$. The defect that moved in the meridian zone had changed side during the observation time, as illustrated in Fig. 3c. The second feature is that two stagnation points are observed on each side of the 320 vesicle. As the vesicles are not spherical, the axis $\mathrm{z}$ is not a symmetry axis so that the stagnation points are not located on this axis, contrarily to what was observed for spherical vesicles sliding along an inclined plane ${ }^{10}$. The position of the stagnation point lies on the median plane $\mathrm{x}=0$ and is located

325 by the angle $\theta$ between the radius of the sphere passing by this point and the axis $\mathrm{z}$ (Fig. 3). The variation of the angle $\theta$ with the vesicle aspect ratio, characterized by the non-dimensional number $\mathrm{L} / 2 \mathrm{R}$, is reported in Fig. 4. The angle $\theta$ starts from 0 for spherical symmetrical vesicles and slowly increases with

$330 \mathrm{~L} / 2 \mathrm{R}$. The increase is much sharper when $\mathrm{L} / 2 \mathrm{R}$ approaches 1 (half-sphere). We did not observe vesicles with a ratio $\mathrm{L} / 2 \mathrm{R}$ greater than 1 (with a contact angle greater than $90^{\circ}$ ), which are likely very unstable due to the large bending energy of the membrane at the contact with the substrate.

${ }_{335}$ When the size of the contact zone increases, the orbits of the streamlines depart more and more from planar circular orbits since no flow is allowed in the contact zone. The streamlines have non trivial non planar shapes, which are difficult to characterize (Fig. 3).

\section{Angular velocities}

The period $\mathrm{T}$ of revolution of a defect on an orbit at the surface of a vesicle was experimentally determined by following the projection of marker trajectories over at least one full orbit. An example of the variation of the coordinates

$345 \mathrm{X}$ and $\mathrm{y}$ of the marker versus time, as deduced from side view imaging of the vesicle, is represented in Fig. 5. These variations are well fitted by a sinusoidal function, suggesting the rotational velocity is constant and equal to the frequency of revolution, $\omega=2 \pi / \mathrm{T}$. Typical variation of $\omega$ on one given 350 orbit as a function of the shear rate $\chi^{\prime}$ is illustrated in Fig. 6. The membrane velocity show several remarkable features. First, the velocity $\omega$ varies linearly with the wall shear rate, as predicted for non adhered vesicles in non bounded shear flow $^{14}$ and observed on non adhered vesicles flowing close to 355 a substrate ${ }^{8,11}$. Moreover, for a given shear rate, the values of $\omega$ increase upon decreasing the relative contact zone $L / 2 R$, i. e. upon modifying the aspect ratio of the vesicle. Finally, the rotational velocity on a given streamline depends on its lateral position on the vesicle surface. In order to illustrate this 360 phenomenon, we measured the rotational velocity on various streamlines located on a single vesicle.

The lateral position of a streamline is characterized in the median plane of the vesicle $(x=0)$ by the ratio $s^{*}=s / s_{0}$, of the curvilinear distance between the stagnation point and the 365 streamline $\mathrm{s}$ to the curvilinear distance between the stagnation point and the vesicle top $(\mathrm{x}=0, \mathrm{y}=\mathrm{R}, \mathrm{z}=0), \mathrm{s}_{0}$, as shown in Fig. 3. Fig. 7 shows the variation of $\omega / \hat{\chi}$ with $\mathrm{s}^{*}$ for vesicles observed either from side or bottom view. Velocities are larger towards the stagnation points (SP). This variation is the 370 contrary to that predicted by Kraus et $\mathrm{al}^{14}$ on vesicles flowing in a non-bounded shear flow, where ellipsoid vesicles display smaller angular velocity towards the SP. Our result is likely due to the fact that the contact zone is motionless. It induces that the angular velocities of the orbits at the proximity of the 375 contact line be small in order to limit the viscous dissipation in the membrane. The difference of angular velocity on the various streamlines reveals an effective strong shear in the membrane, since two adjacent lateral elements of the membrane do not remain adjacent after a full revolution. It is 380 also note worthy that the ratio $\omega / \not{\alpha}$ is greater than 0.5 close to the vesicle SP (also observed in Ref. 11), this value being the angular velocity of a rigid fluid in a non-bounded shear flow.

We infer the two extreme values of the angular velocity, $\omega_{0}$ at the vesicle top and $\omega_{\theta}$ on the SP, by fitting the variation of 385 the angular velocity $*$ with an arbitrary linear function, $\omega / \chi^{\prime}=\left(\omega_{0}-\omega_{\theta}\right) \mathrm{s}^{*}+\omega_{\theta}$, as displayed in Fig. 7. The quantity $\omega_{0}-\omega_{\theta}$ vhthus represents the gradient of velocity upon curvilinear displacement along the vesicle membrane. These three parameters are plotted versus the 390 aspect ratio, $L / 2 R$, in Fig. 8. It clearly appears that $\omega / \tilde{\chi}^{\prime}$ is mainly determined by the vesicle aspect ratio $L / 2 \mathrm{R}$ and is weakly sensitive to the vesicle radius in the studied range. $\omega / \chi^{\prime}$ is constant for $\mathrm{L} / 2 \mathrm{R} \leq 0.8$. For larger contact areas, the velocities sharply decrease and display a smaller difference 395 between the top and the SP.

\section{Discussion}

We described the main features of surface flows of giant lipid vesicles adhering to a substrate. We revealed the existence of fast flows on the surface associated with closed non planar 400 orbits around two stagnation points on each side of the vesicle. We showed that flow is very unlikely to occur in the adhesion zone with the substrate, meaning that the lipids do not bind and unbind at the rotational frequency of the membrane. It should be underlined here that the nature of the 405 contact and thus the dissipation might be different for each kind of adhesion: specific or non-specific. While non-specific adhesion will create an homogeneous contact area where all lipids are subjected to the interaction field with the substrate, specific adhesion implies domains of tight adhesion formed 410 by Ni-lipids and Histidin groups separated by non-adhesive of weakly adhesive areas containing pure DOPC lipids. These DOPC lipids may, in principle, flow around the bound lipids, though such a flow has a high cost in terms of energy of viscous dissipation 24. The observation of local lipid flow 415 requires more sophisticated instruments to be detected. The adhesive zone rather acts as an obstacle to the membrane flow, which therefore induces a velocity gradient. The angular velocity on the orbits indeed increases when the orbits are closer to the SP, showing a strong shearing dissipation in the 420 membrane. However, as far as we know, no theoretical nor numerical description of the surface flows of the vesicle membrane is today available to describe the shape of the streamlines and the total viscous dissipation in the membrane. Similar questions have been addressed in the case of a drop 425 adhered to a wall ${ }^{24,25}$ but streamlines are very different from the ones we observed since recirculation of surface molecules to the internal volume is authorized for drop, whereas lipid molecules can not enter into the inner vesicle volume, or in other terms, the surface divergence of the surface velocity 
430 must equal zero in the case of a lipid vesicle. In the case of a cell, a solid cytoskeleton is bound to the membrane via transmembranar proteins. As the contact zone is fixed and the cytoskeleton is an elastic solid, the whole cytoskeleton is expected to remain fixed over the whole cell. Therefore, no

435 fast flow of the cell membrane is expected. Indeed, side view imaging reveal that small heterogeneities on $\mathrm{CHO}$ cells adhered to a glass substrate under shear flow are motionless ${ }^{26}$. However, a small lipid flow may occur around the transmembranar proteins, associated with a strong viscous 440 dissipation as observed, when a thread is pulled out from a $\operatorname{cell}^{27}$. The decrease in $\omega / \not{\alpha}$ i with the adhesion strength, characterised by the ratio $\mathrm{L} / 2 \mathrm{R}$, could have multiple origins including: i) the fact that the larger the adhesive zone, the more the membrane flow is hindered by this obstacle, ii)

${ }_{445}$ Deviation from the spherical shape has previously been shown to decrease the ratio $\omega / \chi^{\prime}$ as derived from theories describing the dynamics of a vesicle under shear flow ${ }^{12-14}$.

\section{Conclusion}

We used a simple experimental approach to describe the fast 450 surface flows of a lipid membrane of an adhered giant vesicle, which had not been studied yet. Our approach relied on the motion of markers, whose size is of the order of one micron. It is worth underlining that molecular flow may also occur in the contact area which might allow the recruitment of surface 455 receptors towards the membrane adhesion zone. Other experimental tools, such as adapted fluorescence techniques (FRAP, FCS), could be of interest to give a further insight into the molecular flows on adhered cells and vesicles. Moreover, we hope that our work will generate numerical 460 studies in order to better understand the dependence of the streamline shapes and of the membrane viscous dissipation as a function of the radius and of the aspect ratio of the vesicles.

We acknowledge Pr. F. Bruckert for the design of the peptide 465 and the help in the functionalization process, Dr. L. Limozin for RICM experiments, Pr. P. Bongrand for tracking numerical codes and Dr. M. Abkarian for discussions. Part of experiments were performed in Laboratoire de Spectrométrie Physique, UMR5588-University J. Fourier, Grenoble, France.

\section{${ }_{470}$ Notes and references}

+ http://www.physics.emory.edu/ weeks/idl/

1 H. Stone, A. Stroock and A. Ajdari, Annu. Rev. Fluid Mech., 2004, 36, 381
4752 R. Alon, D. A. Hammer and T. A. Springer, Nature, 1995, 374, 539; K-C. Chang, D. F. Tees and D. A. Hammer, PNAS, 2000, 97, 11262

3 H. Goldsmith and J. Marlow, Proc. R. Soc. Lond. B. , 1972, 182, 351

4 T. Fischer and H. Schmid-Schnbein, Blood Cells, 1977, 3, 351; T. Fischer, M. Stöhr-Liesen, and H. Schmid-Schönbein, 1978, Science, 202, 894

5 Chaffey, C. E., H. Brenner, and S. G. Mason, Rheol. Acta., 1965, 4, 64

6 H. Schmid-Schnbein and R. Wells, Science, 1969, 165, 288

7 de Haas, K. H., C. Blom, D. van den Ende, M. G. H. Duits, and J. Mellema, Phys. Rev. E, 1997, 56, 7132-7137

8 M. Abkarian and A. Viallat, Biophys. J., 2005, 89, 1055; Abkarian, M., C. Lartigue, and A. Viallat,. Phys. Rev. Lett., 2002, 88, 81038107

9 B. Lorz, R. Simson, J. Nardi, and E. Sackmann, Europhys. Lett., 2000, 51, 468-474.

10 Abkarian, M., C. Lartigue, and A. Viallat, Phys. Rev. E, 2001, 63, $1-$ 7

11 A. Razpet, G. Gomiscek, V. Arrigler, S. Svetina and B. Zeks, Eur. J. Physiol., 2000, 439 (Suppl),R141-R142

49512 Keller, S., and R. Skalak, J. Fluid Mech., 1982, 120, 27-47

13 T.W. Secomb, and R. Skalak, Q. J. Mech. Appl. Math., 1982, XXXV 2, 233

14 M. Kraus, W. Wintz, U. Seifert, and R. Lipowsky, Phys. Rev. Lett., 1996, 77, 3685

50015 H. Noguchi, and G. Gompper, Phys. Rev. Lett., 2004, 93, 8102

16 S. Sukumaran and U. Seifert, Phys. Rev. E., 2001, 64, 1-11; U. Seifert, Phys. Rev. Lett., 1999, 83, 876-879

17 I. Cantat and C. Misbah, Phys. Rev. Lett., 83, 880-884

16 D. Cuvelier, C. Vézy, A. Viallat, P. Bassereau, and P. Nassoy, J. Phys.: Condens. Matter, 2004, 16, S2427-S2437

17 F. Brochard-Wyart, and P. G. de Gennes, PNAS, 2002; 99;7854-7859

18 R M. C. Dawson, D. C. Elliott, W. H. Elliott, K. M. Jones, in Data for Biochemical Research, ed Oxford University Press, USA, 3 edn., 1986, pp. 409

51019 M. Conti, G.Falini and B. Samorì, Angew Chem Int Ed Engl, 2000, 39, 215-218

20 M. I. Angelova, S. Soléau, P. Méléard, J. F. Faucon, P. Bothorel, Prog. Colloid. Polym. Sci., 1992, 89, 127-131

21 E. Sackmann, R. F. Bruinsma, ChemPhysChem, 2002, 3, 262-269

515 *U. Seifert and R. Lipowsky, Phys. Rev. A, 1990, 42, 4768; R. Lipowky and U. Seifert, Langmuir, 1991, 7, 1867

**] C. Tordeux, J.-B. Fournier, and P. Galatola, Phys. Rev. E, 2002, 65, 041912

22 R. Lipowsky and U. Seifert, Mol. Cryst. Liq. Cryst., 1991, 202, 17-25

52023 O. Sandre O, L. Moreaux L, F. Brochard-Wyart F, PNAS, 1999, 96 (19), 10591-10596

24 C. Pozrikidis, personal communication

25 P., Dimitrakopoulos, and J. J. L., Higdon, J. Fluid Mech., 1997, 336, 351-378

52526 A. Viallat, M. Faivre, M. Abkarian, C. Vézy and N. Glade, presented in part at the Gordon Research Conference colloidal, macromolec and polyelect solutions, Ventura, USA, February, 2004

27 F. Brochard-Wyart, N. Borghi, D. Cuvelier and P. Nassoy, PNAS, 2006, 103, 7660-7663 

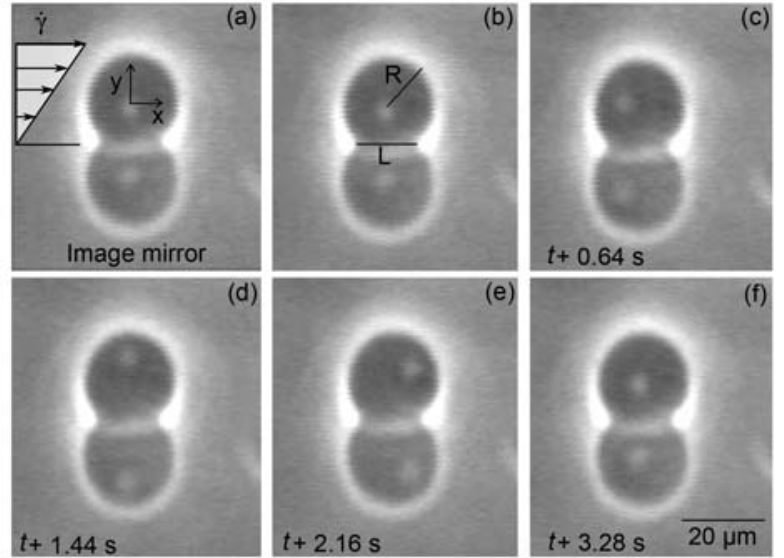

Fig.1 (a) : Side view of a vesicle (Ves4) and its reflection on the substrate subjected to a linear shear flow along the $\mathrm{x}$ direction. (b) : $\mathrm{L}$ is the length of the contact zone. $R$ is the vesicle radius. From (b) to (f): we infer the surface flow from the motion of a lipid defect (clear dot), we used as a marker.

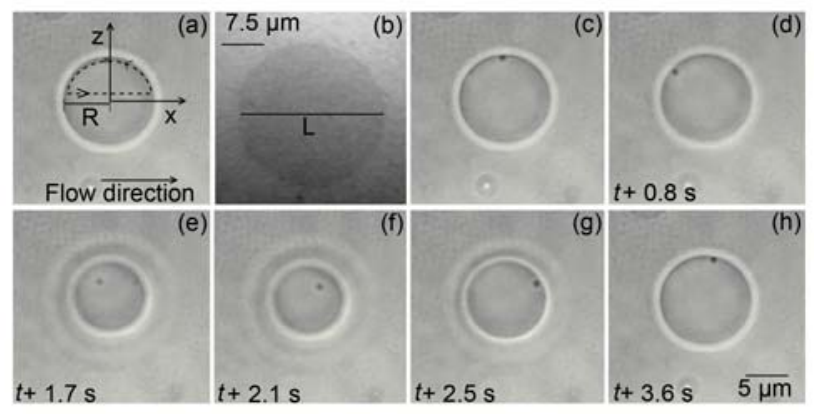

Fig.2 : (a) : Bottom view of Ves5, subjected to a linear shear flow. The dotted line represents the projection of the marker trajectory. (b) : RICM view of ves5 (the scale is different from (a), (c)-(h)). The adhesive zone corresponds to the large homogenous dark disc. (c), (d) and (h): the marker (black dot) avoids the contact zone when approaching the substrate. From (e) to (g) the marker circulates at the vesicle top (the focus varies). 


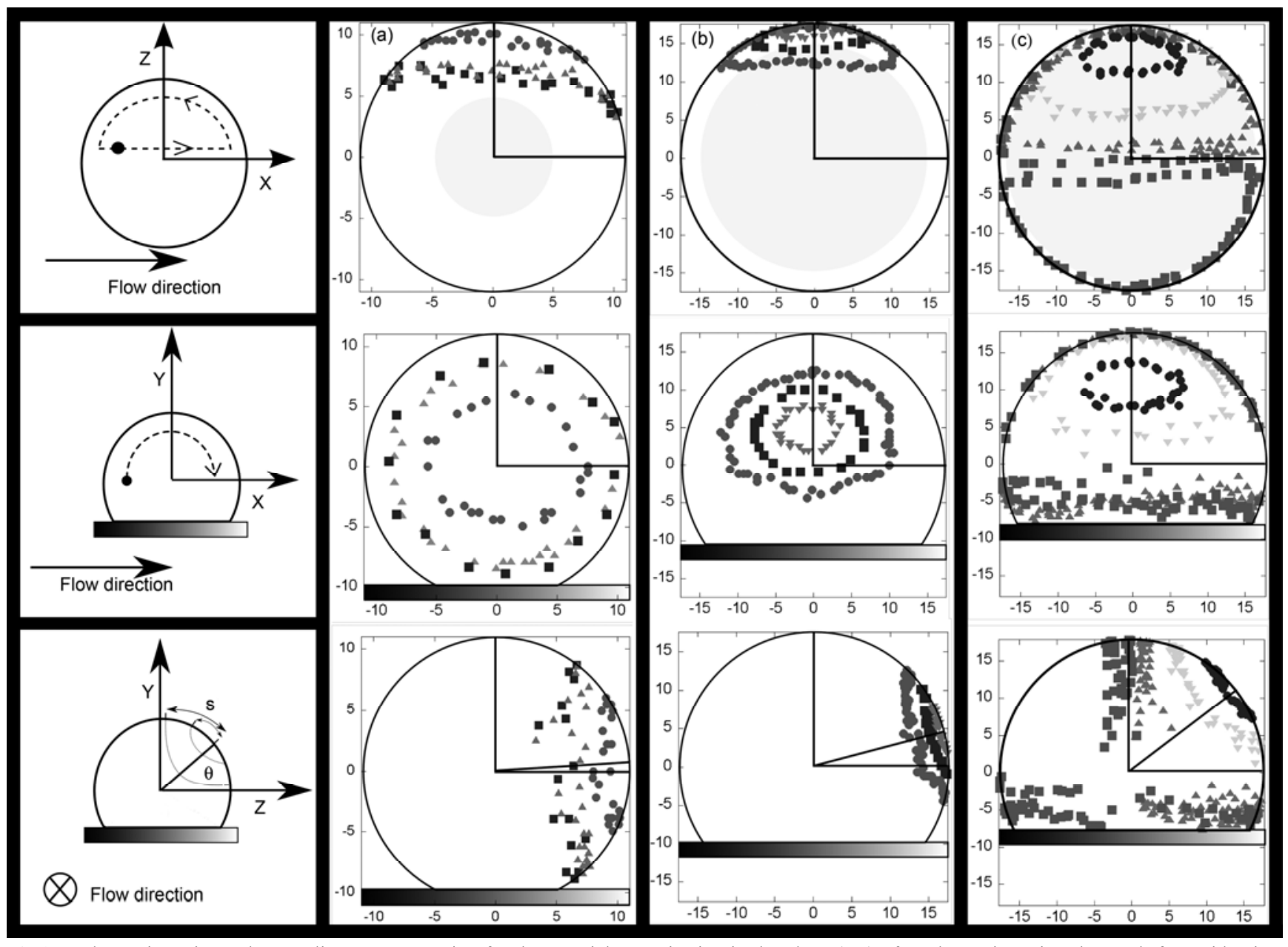

Fig.3 Marker trajectories and streamlines reconstruction for three vesicles. Projection in the plane (x,y) of marker trajectories observed from side view imaging and reconstruction of the corresponding streamlines in $(\mathrm{z}, \mathrm{y})$ and $(\mathrm{x}, \mathrm{z})$ planes : (a) : Ves1; (b) : Ves3. Projection in the plane (x,z) of marker trajectories observed from bottom view imaging and reconstruction of the corresponding streamlines in $(\mathrm{z}, \mathrm{y})$ and $(\mathrm{x}, \mathrm{y})$ planes : (c) : Ves5.

The grey disc in the $(\mathrm{x}, \mathrm{z})$ view represents the contact zone.

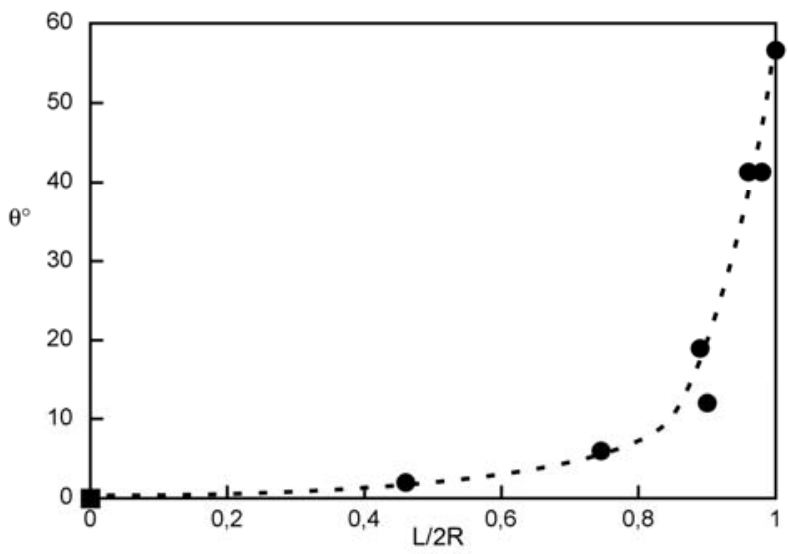

Fig.4 Variation of $\theta$ (defined in Fig. 3) as a function of the aspect ratio $\mathrm{L} / 2 \mathrm{R}$. The dashed line is a guideline for eyes. 


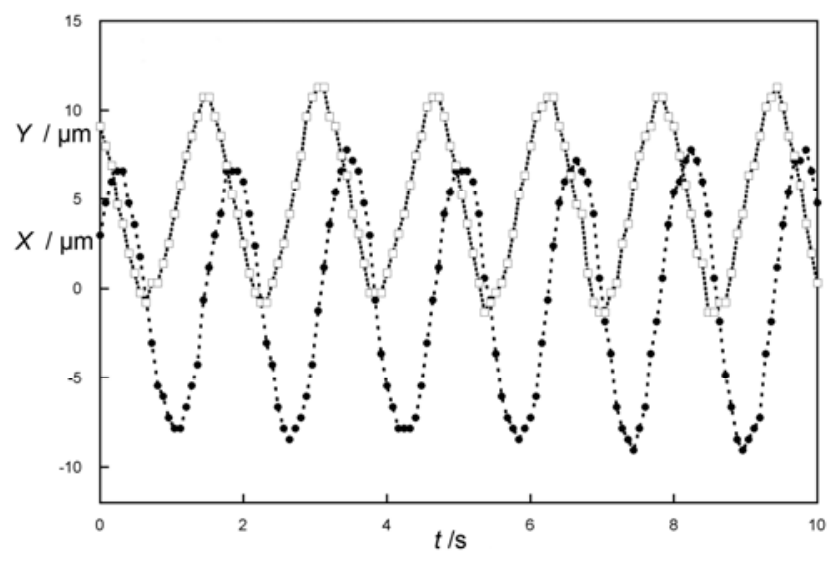

Fig.5 Typical time evolution of the coordinates $\mathrm{x}$ (black circles) and $\mathrm{y}$ (open squares) of a marker on Ves3 as deduced from side view imaging. The lines are guidelines for eyes.

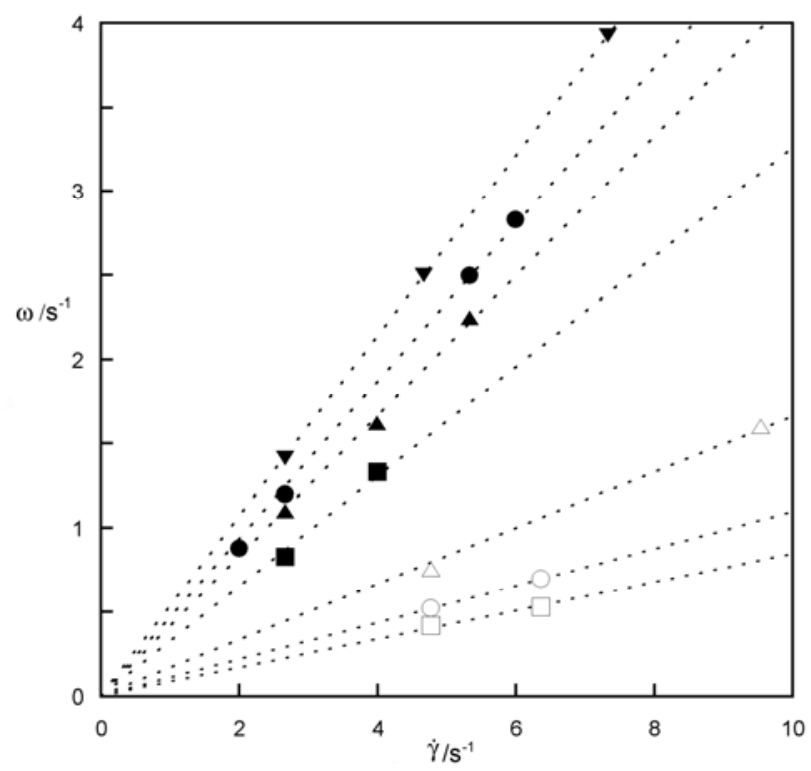

Fig.6 Linear variation of the angular velocity $\omega$ of a circulating marker as a function of the shear rate $\dot{\gamma}$ observed on fixed orbits: $\bullet$ : Ves1; $\mathbf{\square}$ Ves2; $\boldsymbol{\Delta}:$ Ves3; $\boldsymbol{\nabla}:$ Ves4; O : Ves5; $\square:$ Ves6 and $\Delta:$ Ves7. Dashed lines are linear fit. 


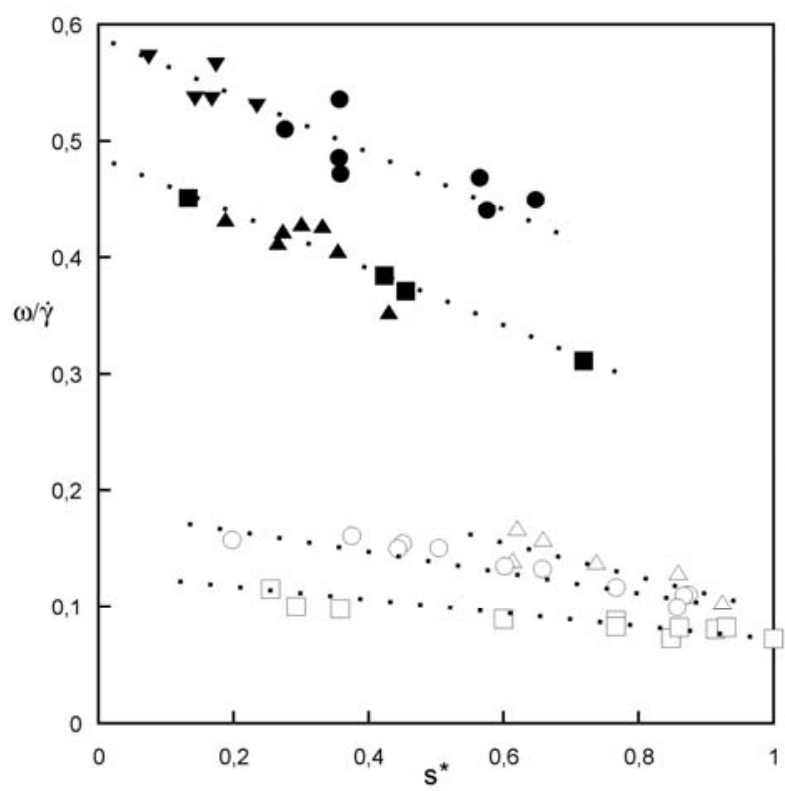

Fig.7 Variation of the reduced angular velocity $\omega / \dot{\gamma}$ of a circulating marker as a function of the dimensionless curvilinear distance $\mathrm{s}^{*}=\mathrm{s} / \mathrm{s}_{0}$. $\bullet$ : Ves1; : Ves2; $\boldsymbol{\Lambda}:$ Ves3; $\boldsymbol{\nabla}:$ Ves4; O : Ves5; $\square:$ Ves6 and $\Delta:$ Ves7. $\dot{\gamma}$ is varied in the range $1.13-11 \mathrm{~s}^{-1}$

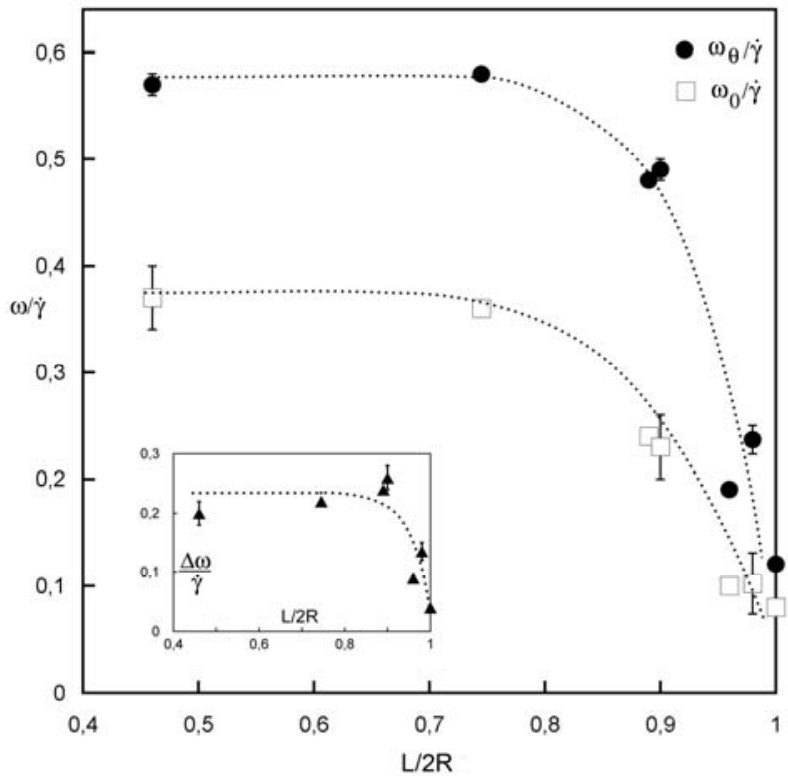

Fig.8 Variation of the reduced angular velocity extrapolated at the stagnation point, $\omega_{\theta} / \dot{\gamma}$, and at the vesicle top, $\omega_{0} / \dot{\gamma}$, versus the aspect ratio $\mathrm{L} / 2 \mathrm{R}$. Insert: variation of the reduced gradient angular velocity as a function of $\mathrm{L} / 2 \mathrm{R}$. Dashed lines are guidelines for eyes. 
Table 1 Experimental parameters for 7 vesicles. $\mathrm{L}$ is the diameter of the contact area, and $\mathrm{R}$ the radius of the vesicles.

$\begin{array}{cccc}\text { Name } & \text { Radius } / \mu \mathrm{m} & \mathrm{L} / 2 \mathrm{R} & \text { Substrate } \\ \text { Ves1 } & 11.0 & 0.46 & \text { Histidin } \\ \text { Ves2 } & 11.0 & 0.89 & \text { Histidin } \\ \text { Ves3 } & 17.5 & 0.90 & \text { Histidin } \\ \text { Ves4 } & 10.9 & 0.74 & \text { Histidin } \\ \text { Ves5 } & 18.4 & 0.96 & \text { Polylysin } \\ \text { Ves6 } & 9.4 & 1.0 & \text { Polylysin } \\ \text { Ves7 } & 11.4 & 0.98 & \text { Polylysin }\end{array}$

